\title{
Cohérence des prévisions et place de l'expertise : les nouveaux défis pour la prévision des crues
}

\author{
Lionel BERTHET ${ }^{(1)}$, Audrey VALÉRY (2), Rémy GARÇON ${ }^{(2)}$, Renaud MARTY(1), Lætitia MOULIN ${ }^{(2)}$, \\ Damien PUYGRENIER ${ }^{(2)}$, Olivier PIOTTE ${ }^{(3)}$, Matthieu LE LAY ${ }^{(2)}$, Bruno JANET ${ }^{(3)}$ et François DUQUESNE(3) \\ (1) DREAL Centre-Val de Loire, service de prévision des crues Loire - Cher - Indre (SPC LCI) - \\ courriels : lionel.berthet@developpement-durable.gouv.fret renaud.marty@developpement-durable.gouv.fr \\ (2) Électricité de France - division technique générale (EDF-DTG) - \\ courriels : audrey.valery@edf.fr,remy.garcon@edf.fr, laetitia.moulin@edf.fr,damien.puygrenier@edf.fret mathieu.le-lay@edf.fr \\ (3) Service central d'Hydrométéorologie et d'Appui à la Prévision des Inondations (MTES/DGPR/SCHAPI) - \\ courriels : olivier.piotte@developpement-durable.gouv.fr,bruno.janet@developpement-durable.gouv.fr et francois.duquesne@developpement-durable.gouv.fr
}

\begin{abstract}
RÉSUMÉ. - De nombreux outils mis en œuvre par les prévisionnistes de crues ont d'abord été conçus pour réaliser des prévisions fiables et précises en un point et à un instant de prévision. Or, les utilisateurs demandent des prévisions de plus en plus nombreuses : plus fréquentes et pour davantage de sites. Dès lors, assurer le bon degré de cohérence des prévisions, tout en maintenant la qualité de chaque prévision, devient un enjeu fort pour les équipes de prévisionnistes. La cohérence des prévisions englobe plusieurs aspects : (i) la cohérence spatio-temporelle des prévisions faites à un instant donné, (ii) le respect de la structure temporelle de chaque prévision (pour différents horizons) et (iii) la cohérence des prévisions successives au cours d'un événement de crue. L'analyse porte sur deux organismes qui ont des contextes et des objectifs différents en matière de prévision des crues : EDF et le réseau Vigicrues. Leurs équipes travaillent avec des premiers outils qui demandent encore un effort de développement, voire de recherche appliquée. Outre le défi technique, cette augmentation de la demande impose de rechercher un équilibre maîtrisé entre l'automatisation des chaînes de prévision et la plus-value de l'expertise humaine. Les premières réflexions sur la cohérence des prévisions soulignent l'importance de l'analyse et de l'interprétation humaine, et la nécessité de bien positionner le prévisionniste dans la chaîne de prévision.
\end{abstract}

Mots-clés : prévision des crues, défis opérationnels, cohérence temporelle, cohérence spatiale, expertise humaine, systèmes opérationnels

\section{Forecasts consistency and human assessment: what are the next challenges for operational flood forecasting?}

\begin{abstract}
Many operational tools used in France by flood forecasters were first designed to achieve reliable and accurate forecasts at a given location and at a given forecast starting time. Yet, users ask for more frequent forecasts and for more locations. Thus, ensuring the right consistency degree of the forecasts (still maintaining the quality of each forecast taken individually) became recently a major challenge for operational forecasting services. The notion of consistency encompasses several properties: (i) the spatio-temporal consistency of the forecasts issued at a given time on a river network, (ii) the right temporal structure of the forecast for the different lead times and (iii) the consistency of the successive forecasts during a flood event. The analysis focuses on two organizations with different flood forecasting contexts and objectives: the French electricity supplier EDF and the French public Flood Forecasting Services ("Vigicrues network"). Their forecasters teams are currently working with first tools in order to improve these consistencies. Some of these tools integrate the consistency issue into the hydrometeorological modelling while others facilitate the consideration of this issue in the forecaster's analysis. More applied research and development are still required. In addition to the technical challenge, increasing the number of forecasts issued calls for a controlled balance between the automation of the operational forecasting chain and the gain due to human expertise. Early reflections on forecasts consistency emphasize the utter importance of the human assessment and interpretation in order to achieve good forecasts and the need to position the forecaster well within the operational forecasting organization.
\end{abstract}

Key-words: flood forecasting, operational challenges, spatial consistency, temporal consistency, human expertise, operational systems

\section{INTRODUCTION}

Dans de nombreux pays, des équipes des services publics ou d'entreprises du secteur privé établissent régulièrement des prévisions de débit ou de cotes de cours d'eau. Les centres de prévision d'EDF - DTG et le réseau Vigicrues, qui rassemble les services de l'État en charge du suivi et de la prévision sur les cours d'eau, comptent parmi les acteurs majeurs de la prévision hydrométéorologique en France en matière de compétences opérationnelles et de volumes de prévisions. Bien que leurs objectifs finaux soient multiples et pour partie différents (gestion de crise institutionnelle et 
information du public, sûreté des grands ouvrages hydrauliques et optimisation de l'usage de la ressource en eau), les chaînes opérationnelles développées par EDF et, plus récemment, par le réseau Vigicrues visent un objectif commun : fournir une prévision fiable et la plus riche possible à des décideurs en différents points d'un réseau hydrographique. Pour atteindre cet idéal, les équipes opérationnelles de ces deux organismes ont fait, comme beaucoup d'autres, le choix d'estimer et de diffuser une information quantitative sur l'incertitude associée aux prévisions.

Bien que cet objectif ne soit pas encore pleinement atteint, nous constatons une augmentation forte de la demande en prévisions par les gestionnaires de crise ou par les gestionnaires d'ouvrages hydrauliques. Cette demande quantitative porte sur (i) le périmètre couvert (accroître nombres de bassins versants suivis et de points de prévision), (ii) la fréquence (passer d'une prévision quotidienne à plusieurs) et (iii) la résolution temporelle (privilégier les pas de temps fins). Y répondre est un défi pour les prochaines années, qui devra être relevé en maintenant la qualité des prévisions (autre attente forte des utilisateurs), voire en l'améliorant (par exemple, des progrès sont nécessaires sur la fiabilité de l'estimation de l'incertitude prédictive), mais aussi en la diffusant au moment le plus opportun pour les utilisateurs.

Or nos outils de prévision ont été initialement pensés dans le but de fournir une prévision en un point et à un instant donnés : leur conception conduit à estimer les prévisions en grande partie indépendamment pour chaque site et chaque instant de prévision.

Par ailleurs, les systèmes de production placent la prévisionniste $^{1}$ au cœur de la chaîne opérationnelle. Cette dernière reste indispensable pour contrôler la qualité des données utilisées et le bon fonctionnement des modèles, mais également pour accroître la qualité des prévisions en apportant sa compréhension des phénomènes hydrométéorologiques en cours et du comportement des modèles, et enfin pour porter et expliquer les prévisions auprès des utilisateurs.

Nos systèmes opérationnels de prévision évoluent régulièrement, que ce soit celui d'EDF ou celui du réseau Vigicrues (qui généralise actuellement la diffusion de prévisions quantitatives accompagnées d'une estimation de leurs

1. Les équipes de prévisionnistes étant mixtes, « la » ou « le » prévisionniste seront cités indifféremment. incertitudes), pour intégrer les avancées scientifiques et améliorer la productivité. Deux enjeux, communs à nos équipes, sont à prendre en compte pour répondre aux attentes d'une augmentation du nombre de prévisions fiables élaborées :

1. assurer la cohérence spatio-temporelle des prévisions, qui est une propriété recherchée sur le plan technique et un facteur de lisibilité des prévisions et donc de crédibilité pour les utilisateurs ;

2. optimiser le rôle du prévisionniste au sein de la châ̂ne opérationnelle, l'adapter aux évolutions contextuelles et améliorer la productivité.

\section{CHAÎNES OPÉRATIONNELLES ET ÉSTIMATION DES INCERTITUDES}

Pour fournir une estimation fiable de l'incertitude totale des prévisions, nos chaînes opérationnelles prennent en compte les deux sources d'incertitude qui contribuent le plus fortement à l'incertitude prédictive [Krzysztofowicz, 1999] : l'incertitude météorologique et l'incertitude de modélisation (hydrologique ou hydraulique). Tandis que les prévisionnistes d'EDF enchaînent des prévisions météorologiques et hydrologiques [Moulin et al., 2018], les prévisionnistes du réseau Vigicrues se concentrent sur les prévisions hydrologiques et hydrauliques.

\section{II.1. Les composantes météorologiques}

La chaîne opérationnelle d'EDF (Duan et al., 2016) intègre les prévisions météorologiques d'ensemble émises par le CEPMMT (51 membres, prévisions à 14 jours) pour rendre compte de l'incertitude sur les précipitations et températures futures. L'ensemble est " mélangé » par ré-échantillonnage à des prévisions par analogues hydrométéorologiques pour en corriger la sous-dispersion [Courbariaux, 2017], et pallier ainsi le manque de fiabilité statistique des modèles bruts. Cette étape est réalisée par le prévisionniste sur la base de son analyse de la situation météorologique et de l'examen des sorties de plusieurs modèles (figure 1). Elle permet d'obtenir des scénarios (trajectoires) équiprobables formant un ensemble calibré (non sous-dispersif).

Les prévisionnistes du réseau Vigicrues se basent principalement sur les prévisions expertisées par Météo-France (aux

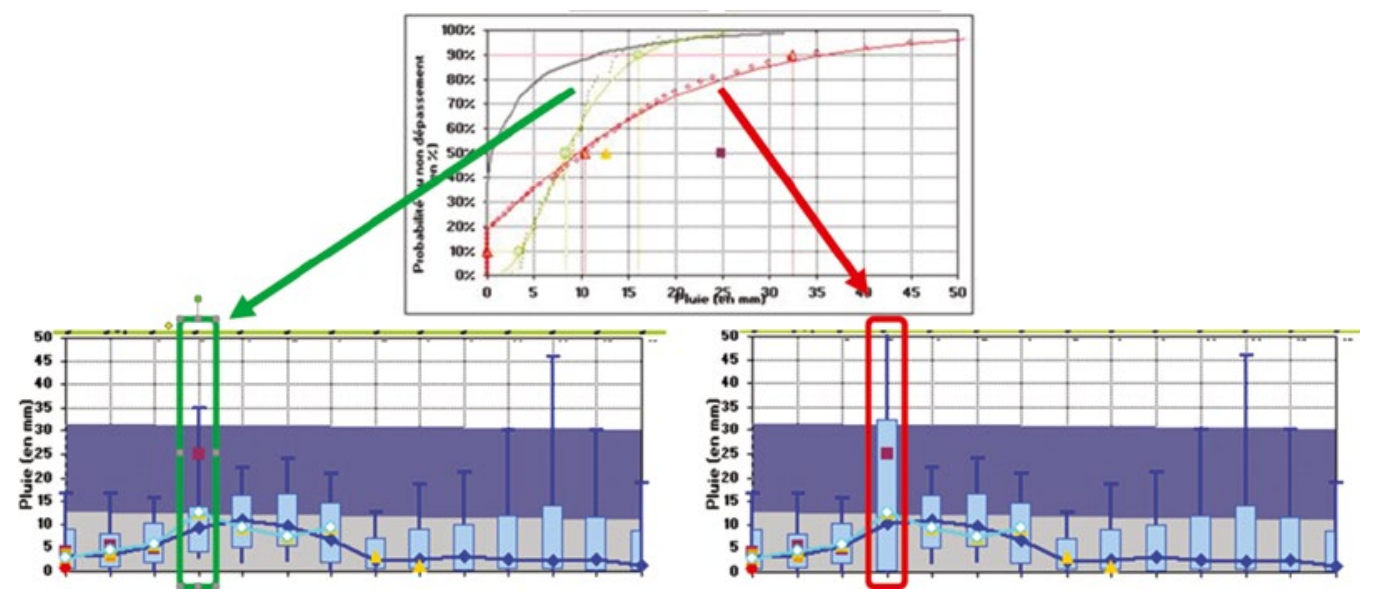

Figure 1 : Exemple d'expertise EDF de la distribution des pluies journalières. Le graphique du haut présente la distribution des 51 membres de la $4^{e}$ échéance brute en vert et expertisée en rouge; les deux graphiques du bas permettent de comparer les distributions de pluies à la $4^{e}$ échéance avant expertise (en bas à gauche), et après expertise (en bas à droite). 
pas de temps de 3 et 24 heures). L'incertitude sur la composante météorologique est estimée par les prévisionnistes de Météo-France qui la traduisent par des intervalles (non calibrés) encadrant les lames d'eau prévues sur 24 heures, par bassin versant ou localement, à partir desquels est créée une collection de scénarios alimentant les modèles hydrologiques. Cela soulève plusieurs difficultés pour l'analyse par le prévisionniste dont (i) l'absence d'estimation a priori de la vraisemblance de chacun de ces scénarios, et, par conséquent, la fiabilité de cette collection et (ii) la dépendance de la temporalité des prévisions hydrologiques à un seul scénario « source ». Le SCHAPI et plusieurs SPC travaillent actuellement à l'intégration de prévisions météorologiques d'ensemble.

Dans les deux cas, l'expertise humaine vise à assurer un certain degré de cohérence spatiale des données de précipitation et de température prises en compte dans la suite du processus.

\section{II.2. Les composantes hydrologiques et hydrauliques}

Les services de prévision des crues (SPC) de l'État disposent de différents modèles hydrologiques et hydrauliques, tous déterministes. L'incertitude prédictive (incertitude de modélisation) est estimée à l'aide du post-traitement statistique QUOIQUE développé et implémenté dans l'outil OTAMIN par l'IRSTEA [Bourgin, 2014 ; Viatgé et al., 2018]. Comme la plupart des post-traitements, cette méthode est appliquée indépendamment à chaque site de prévision, à chaque instant de prévision (exécution du modèle) et pour chaque horizon de prévision. La prévisionniste peut intervenir à différentes étapes de la chaîne de production ; elle contribue en particulier en sortie de la chaîne de calculs en "retravaillant » les résultats du post-traitement pour exprimer sa compréhension de l'événement hydrométéorologique et du fonctionnement des modèles. Les prévisions hydrologiques sont communiquées sur le site Vigicrues (www.vigicrues.gouv.fr) sous la forme de trois tendances (basse, centrale et haute) correspondant à des quantiles au non-dépassement de la variable prévue (débit ou hauteur) à différents horizons.

EDF s'appuie sur le modèle hydrologique MORDOR déterministe semi-distribué [Garavaglia et al., 2017]. Bien que le modèle ait été initialement conçu pour représenter les flux entre les différents compartiments hydrologiques au sein de chaque bassin versant indépendamment, une cohérence régionale a été recherchée lors de la calibration des paramètres par bassin. Une version spatialisée du modèle MORDOR existe [Rouhier et al., 2017] mais n'a pas encore été intégrée à la chaine opérationnelle. L'incertitude de modélisation est également estimée par un post-traitement basé sur une description empirique des erreurs [Mathevet, 2010]. Le prévisionniste peut encore retoucher la prévision en sortie de la chaîne. Les prévisions sont communiquées sous forme d'ensembles (scénarios cohérents correspondant à des trajectoires possibles), de leurs synthèses, ou encore d'informations sur les probabilités de dépassement de seuils pour répondre aux différentes attentes des utilisateurs internes (optimiseurs de la production et gestionnaires d'ouvrages en charge de la sûreté).

Les deux chaînes de prévision présentent plusieurs points communs : elles sont basées sur des modèles hydrologiques ou hydrauliques déterministes dont les sorties subissent des post-traitements basés sur des approches similaires (post-traitements empiriques " calibrés 》 pour assurer la fiabilité de l'estimation de l'incertitude). Par ailleurs, l'expertise de la prévisionniste, en particulier celle intégrée en sortie de chaîne, n'est pas neutre, relativement à la question de la cohérence des prévisions. Enfin, EDF et le réseau
Vigicrues étudient actuellement des premières solutions visant à améliorer intrinsèquement la cohérence spatio-temporelle des prévisions.

La différence principale entre les deux chaînes opérationnelles réside dans l'utilisation d'ensembles par EDF, dont chaque membre (scénario) présente une structure temporelle (aux différents horizons de prévision) plausible respectant la dynamique des phénomènes hydrométéorologiques, tandis que le réseau Vigicrues s'appuie, pour décrire l'incertitude prédictive, sur une collection de " tendances » (quantiles de débits en fonction des horizons) qui ne constituent pas des trajectoires vraisemblables.

\section{PISTES TECHNIQUES IDENTIFIÉES POUR AMÉLIORER LES COHÉRENCES DE NOS PRÉVISIONS}

L'objectif ici est d'évoquer les pistes identifiées à ce jour pour améliorer les cohérences de nos prévisions. Ces pistes sont à l'étude et toutes ne constituent pas des solutions déjà choisies par nos équipes. Un travail important de développement, voire de recherche appliquée en lien avec la communauté scientifique, reste nécessaire pour tirer le meilleur parti de ces premières idées.

\section{III.1. «Cohérence » ou « cohérences » des prévisions ?}

La notion de " cohérence de prévision » recouvre en réalité plusieurs propriétés distinctes. Nos équipes recherchent les cohérences suivantes :

1. La cohérence spatio-temporelle est, pour un instant de prévision donné, le respect de la dynamique de la crue d'amont en aval et aux confluences, par les prévisions aux différents points du réseau hydrographique.

2. Toujours à un instant de prévision donné, la cohérence temporelle de l'estimation de l'incertitude prédictive aux différents horizons (pour une prévision en un lieu donné) est un enjeu, notamment car les post-traitements appliqués aux sorties de modèles déterministes sont «calibrés » indépendamment pour chaque horizon de prévision.

3. Enfin, une seconde cohérence temporelle est recherchée d'une prévision à la suivante. Par ailleurs, la cohérence des estimations successives de l'incertitude prédictive est un enjeu en soi : des écarts forts peuvent inquiéter les utilisateurs qui s'attendent à ce que l'incertitude soit peu à peu réduite.

\section{III.2. Quelles pistes pour améliorer la cohérence spatio-temporelle des prévisions ?}

La cohérence spatio-temporelle des entrées des modèles hydrologiques est un pré-requis pour assurer la cohérence des prévisions hydrologiques. Dans un monde idéal, un unique modèle météorologique, parfait, serait utilisé et la cohérence des prévisions météorologiques serait garantie par sa cohérence intrinsèque. Dans le monde réel, plusieurs modélisations météorologiques sont employées et les apports de l'expertise humaine peuvent rompre cette cohérence. D'autres moyens doivent alors être mis en œuvre pour tenter d'assurer ces différentes cohérences.

Au-delà, la conception des « chaînes » de prévision (dans lesquelles un ou plusieurs modèles hydrauliques succèdent à un ou plusieurs modèles hydrologiques) suggère de propager les incertitudes en combinant, à chaque étape, les deux sources principales d'incertitude : l'incertitude des données 
d'entrée (résultant de modèles amont) et l'incertitude de modélisation ; il s'agit d'une généralisation de l'approche proposée par Krzysztofowicz [1999].

Pour assurer la cohérence spatio-temporelle de l'ensemble de la chaîne, la structure de corrélation spatio-temporelle doit être prise en compte ou reconstruite à chaque étape. Cette combinaison peut se faire " classiquement » en propageant un ensemble de trajectoires et en lui appliquant un post-traitement empirique pour obtenir un ensemble fiable (calibré) à l'issue de chaque étape. Si ce dernier brise la structure spatio-temporelle de l'ensemble, il est alors nécessaire de la " reconstruire " pour que l'ensemble pris en entrée du modèle suivant comporte des trajectoires plausibles. Une approche par tirages dans la distribution prédictive est d'ores et déjà employée par EDF : ces tirages sont « ré-ordonnés » en tenant compte de la structure temporelle de l'ensemble avant post-traitement pour définir un ensemble ayant la dispersion de l'ensemble post-traité et la structure temporelle avant post-traitement [Schefzik et al., 2013]. Une méthode similaire, se basant sur une autre source d'information comme les analogues hydrométéorologiques [Bellier et al., 2017], ou des approches plus fondamentalement différentes doivent être étudiées et comparées.

Pour gérer la cohérence spatio-temporelle lors de la phase d'expertise finale aboutissant à la validation des prévisions avant diffusion, plusieurs pistes sont envisagées. La première est d'accompagner le prévisionniste sur l'expertise des prévisions de chaque site en lui permettant d'afficher la prévision et l'incertitude associée qu'il a expertisées sur un ou plusieurs sites liés (par exemple amont), ou encore un " proxy » qui serait une combinaison simple des prévisions probabilistes expertisées amont (figure 2). Au-delà, il est proposé d'organiser l'expertise par «paniers » de bassins versants pour lesquels les outils mis en œuvre par la prévisionniste assureraient, sauf décision contraire de sa part, la cohérence spatio-temporelle de ses actions ; ces outils pourraient notamment être employés pour l'expertise des scénarios météorologiques.

\section{III.3. Quelles pistes pour améliorer la cohérence temporelle de l'estimation de l'incertitude prédictive par post-traitement ?}

Comme nous l'avons vu dans la section précédente, les post-traitements empiriques actuels «brisent » la cohérence temporelle des estimations de l'incertitude prédictive : on passe de trajectoires plausibles sur le plan hydrologique (qu'elles soient déterministes ou d'ensemble) à une série de quantiles en fonction de l'horizon («tendances »). Cette question est très importante pour le réseau Vigicrues, d'une part car le pas de temps principal des prévisions est le plus souvent fin (prévisions horaires, voire infra-horaires) et d'autre part car l'estimation de l'incertitude prédictive résulte, pour le moment, uniquement du post-traitement d'une prévision déterministe (et non des membres d'un ensemble comme pour les prévisions réalisées par EDF).

Une réponse opérationnelle immédiate réside dans la mise à disposition dans la trousse à outils du prévisionniste de fonctions de lissage des tendances qui décrivent l'incertitude prédictive. Le logiciel d'expertise graphique des prévisions EXPRESSO (figure 2) à la disposition des prévisionnistes du SPC Loire - Cher - Indre inclut un tel outil. Construire un algorithme de lissage basé sur la structure temporelle (en fonction de l'horizon) de l'incertitude prédictive permettrait d'aller plus loin.

À plus long terme, la démarche de reconstruction de trajectoires présentant une cohérence temporelle, décrite dans la section précédente pour assurer la cohérence spatio-temporelle, répond également au besoin de cohérence de l'estimation de l'incertitude prédictive entre horizons. D'autres approches s'éloignant bien plus du schéma global actuel de nos chaînes opérationnelles sont possibles et ne doivent pas être négligées. Un exemple en est la méthode proposée par Barbetta et al. [2017] pour estimer la distribution jointe de l'incertitude prédictive pour l'ensemble des horizons de prévision directement dans le cadre du post-traitement d'une prévision d'un modèle déterministe.

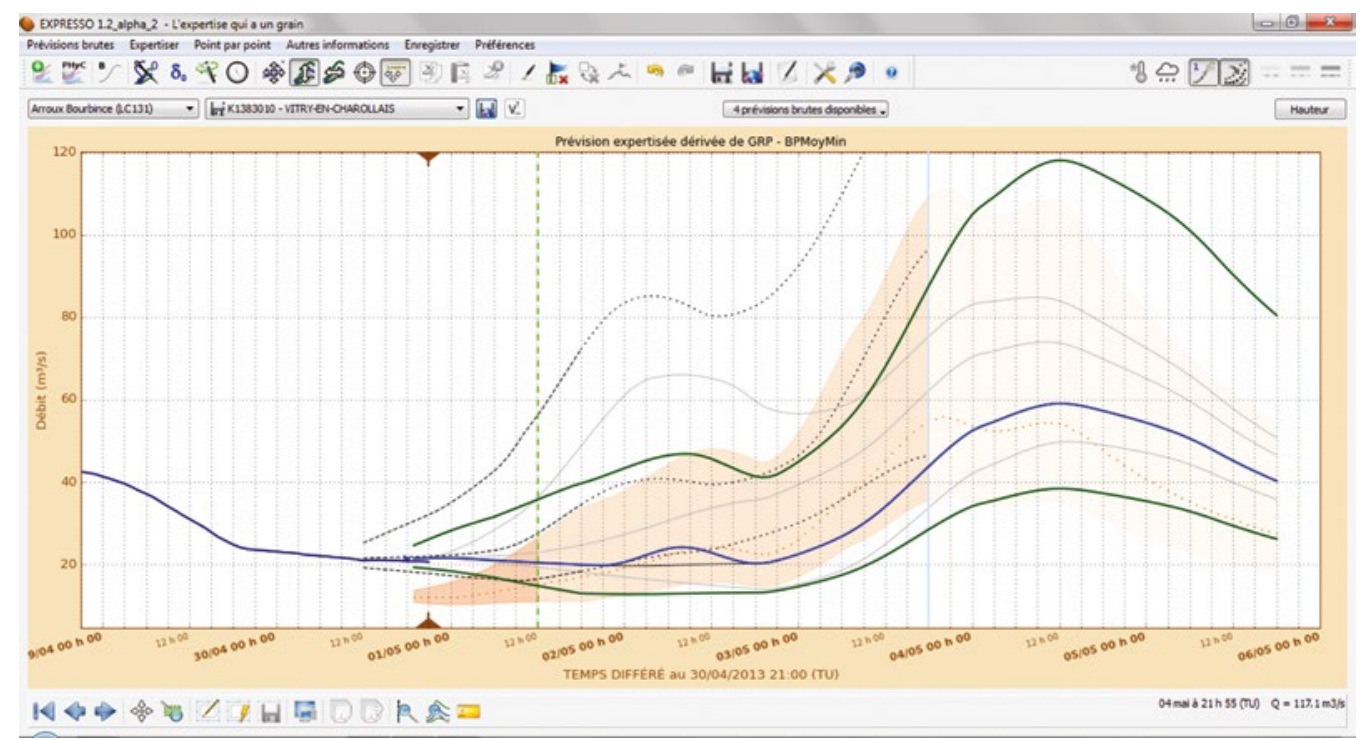

Figure 2 : Expertise à Vitry-en-Charollais (Bourbince, $819 \mathrm{~km}^{2}$ ) le $1^{\mathrm{er}}$ mai 2013. Le prévisionniste construit sa prévision (lignes continues bleue et vertes : quantiles prédictifs associés aux probabilités 0,1,0,5 et 0,9) sur la base des prévisions " brutes" établies par le modèle GRP pour 4 scénarios météorologiques (lignes grises continues), de la prévision validée la veille à 12 h (lignes noires pointillées) et d'un "proxy » correspondant à la prévision en cours d'expertise à Ciry-le-Noble (à l'amont, $343 \mathrm{~km}^{2}$ ), multipliée par 1.5 (aplats et ligne pointillée orangés). 


\section{III.4. Quelles pistes pour améliorer la cohérence temporelle d'un instant de prévision au suivant?}

Les prévisionnistes sont avant tout dépendants du degré de cohérence des prévisions météorologiques. Les prévisionnistes d'EDF comme ceux de Vigicrues sont régulièrement confrontés à des changements forts d'une prévision météorologique à la suivante ${ }^{2}$. Ils font alors face à un dilemme entre la valorisation des dernières informations (assimilées par le modèle météorologique) et un effet de " yoyo » sur des variations brutales d'une prévision à l'autre (figure 3 ).

Dans certains cas, l'expertise des prévisionnistes d'EDF peut rectifier en partie ces évolutions, tout en veillant à profiter au mieux du rafraîchissement du modèle. Les prévisionnistes

2. Ces inflexions peuvent être la traduction d'éléments nouveaux entrant dans l'initialisation des modèles, dont la prise en compte est légitime, mais sont parfois dues en partie à des artefacts numériques, à des choix de modèles différents par le prévisionniste météorologue (dont les comportements peuvent diverger dans certaines circonstances) ou encore aux rotations des équipes de prévisionnistes. du réseau Vigicrues, confrontés à une telle difficulté, dialoguent avec leurs partenaires de Météo-France pour analyser les raisons de ce manque de cohérence et le cas échéant adapter le choix des scénarios d'entrée des modèles pluie-débit.

Au-delà, une piste à étudier serait d'incorporer les prévisions des $n$ dernières exécutions des modèles afin de construire et d'expertiser un lissage temporel des $n$ prévisions. Météo-France, confronté aux mêmes enjeux de cohérence temporelle sur la prévision météorologique, travaille à cette approche appliquée au modèle Arome PI, en proposant au prévisionniste les résultats des $n$ dernières exécutions.

Par ailleurs, il convient de maîtriser l'apport de la subjectivité humaine dans l'élaboration des prévisions, en particulier lors des changements d'équipes de prévisionnistes (figure 4). L'affichage des dernières prévisions expertisées (ou non expertisées), dans les outils d'expertise (figure 2) est une première réponse permettant au prévisionniste d'en tenir compte dans son expertise sans minimiser l'information apportée par les dernières observations (assimilation de données).
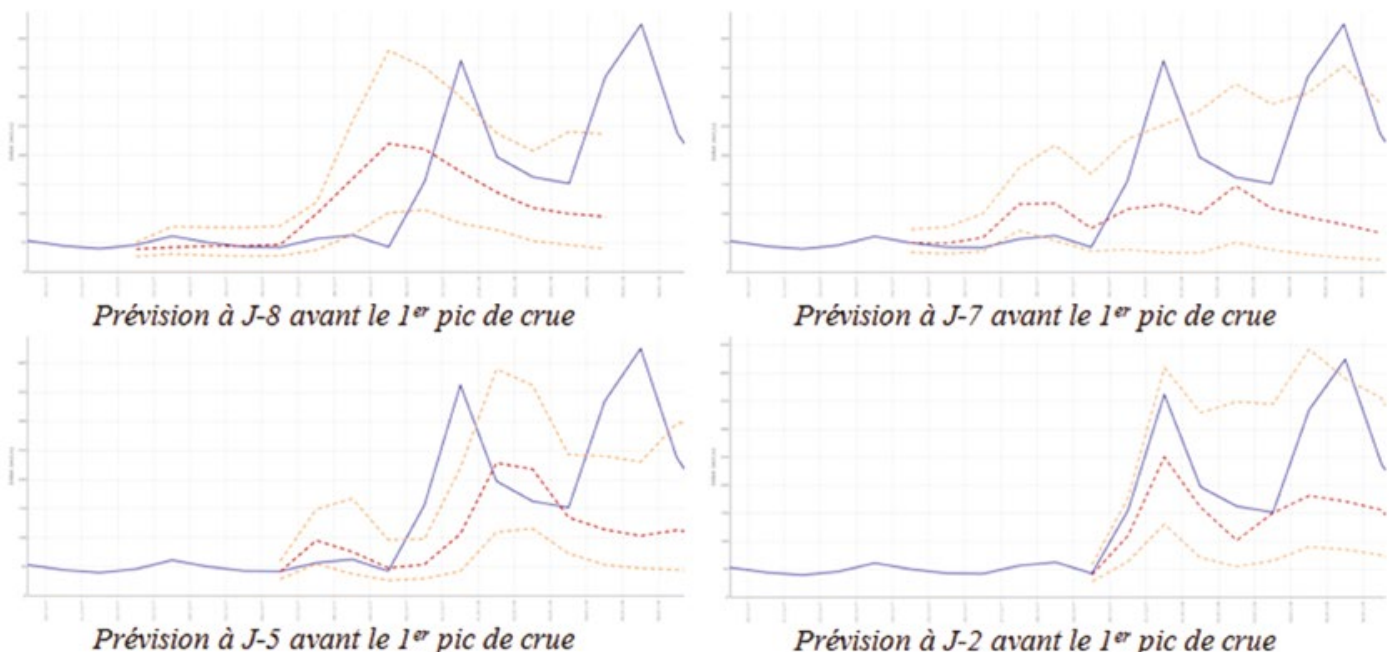

Prévision à J-7 avant le $1^{\text {er }}$ pic de crue

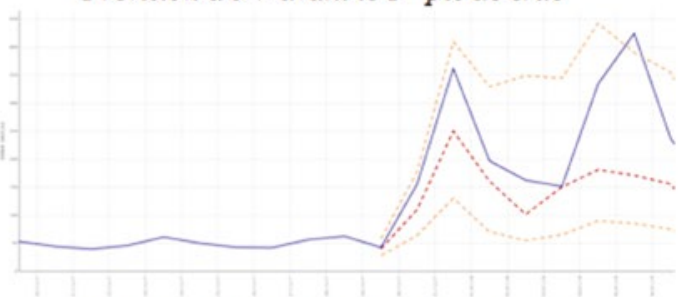

Prévision à J-2 avant le $1^{\text {er }}$ pic de crue

Figure 3 : Prévisions établies pour les crues de l'Ain de décembre 2017. Assurer la cohérence des prévisions entre elles est un défi de taille. Lignes bleues : les débits observés; lignes pointillées orange et rouges : les quantiles de débit prévu.

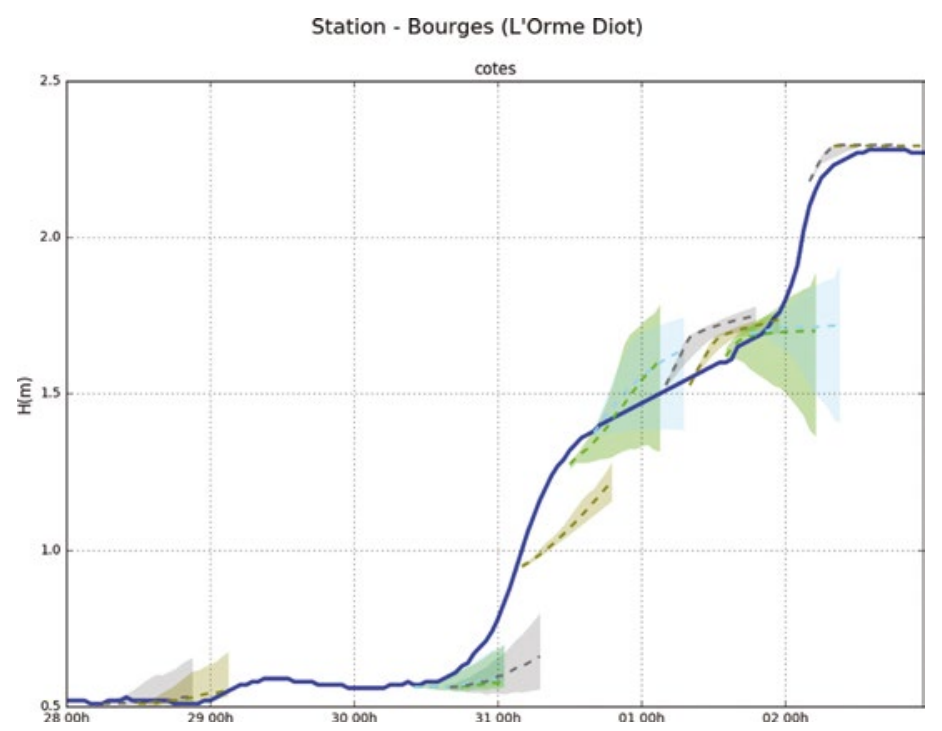

Figure 4 : Prévisions successives (faisceaux) réalisées sur l'Auron à Bourges [Orme Diot]. Deux équipes de prévisionnistes se sont succédé au cours de l'événement. On distingue assez nettement les passages de relais : une équipe choisissait de fournir des estimations de l'incertitude prédictive bien plus faibles que l'autre. 


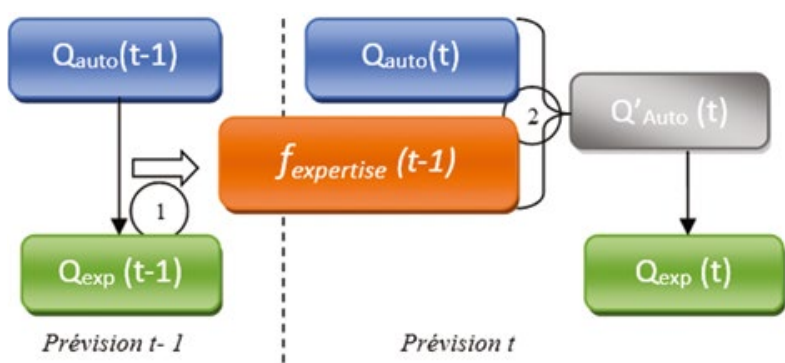

Figure 5 : Processus de valorisation de la dernière expertise. (1) Déformation $f$ de la prévision automatique en prévision expertisée à $t-1$ et (2) application de f sur la prévision automatique à t pour en déduire une prévision automatique "valorisée ».

Une piste intéressante (figure 5) serait de valoriser l'expertise apportée lors de la précédente prévision hydrologique diffusée par un prétraitement de la prévision automatique en cours, avant un geste final d'expertise par la prévisionniste.

On peut noter que l'analyse de la cohérence des prévisions d'un cycle de production au suivant est aussi une opportunité : l'utilisation de la persistance du signal dans l'analyse des prévisions peut améliorer la prise de la décision optimale ou la diffusion d'alerte. C'est d'ailleurs un des principes mis en œuvre par le système européen EFAS [Ramos et al., 2007].

\section{IV. ÉVOLUTIONS DU RÔLE DES PRÉVISIONNISTES DANS L'ÉLABORATION DES PRÉVISIONS}

La mission actuellement confiée aux prévisionnistes est double : ils sont d'abord chargés de l'élaboration des prévisions, mais également de leur portage auprès de certains utilisateurs avec lesquels ils interagissent et accompagnent dans l'emploi des prévisions. Dans cette partie, l'évolution du rôle du prévisionniste dans la chaîne opérationnelle de prévision sera discutée, tandis que certaines conséquences sur le portage et la bonne utilisation des prévisions seront identifiées dans la suivante.

\section{IV.1. Vers la mise en place de « cycles " automatisés de prévision?}

De façon générale pour optimiser la production de prévisions, et plus spécifiquement pour faire face à l'augmentation de la demande de prévisions (dans une durée qui ne peut pas être étendue), renforcer l'automatisation des chaînes opérationnelles est une piste évidente [Pagano, 2013] permettant d'alléger le prévisionniste de certaines opérations et de les mener en "tâches de fond » et plus rapidement. L'exemple des principaux centres météorologiques à travers le monde, qui mettent en œuvre quotidiennement des chaînes de prévision automatisées, vient immédiatement en tête. Les prévisions qui en résultent sont largement diffusées. Pour autant, les prévisions météorologiques expertisées par des prévisionnistes, basées sur les sorties automatiques, continuent d'apporter une véritable plus-value et sont employées par de nombreux utilisateurs.

Il est cependant périlleux de concevoir les chaînes de prévision hydrologique uniquement en les calquant sur les organisations des centres de prévision météorologique. Plusieurs différences majeures limitent la portée de l'analogie. Tout d'abord, le système physique étudié est très différent : tandis que la météorologie s'intéresse à un système physique unique (qu'on peut résumer « au premier ordre » à l'atmosphère), les centres de prévision hydrologique travaillent sur plusieurs systèmes indépendants (les bassins versants). La modélisation de l'atmosphère se fonde en grande partie sur les lois de la mécanique des fluides, équations "générales » en ce qu'elles sont applicables en tout point, tandis que notre compréhension des phénomènes d'écoulement reste plus limitée et les représentations que nous en faisons (les modélisations hydrologiques et, dans une moindre mesure, hydrauliques) sont plus variées et «adaptées » aux bassins versants. Par ailleurs, si les modèles météorologiques assimilent un très grand nombre de données pour déterminer leur état initial (du système unique qu'est l'atmosphère), les modélisations hydrologiques requièrent très peu de données d'entrée : le contrôle de leur pertinence est un enjeu majeur pour obtenir des prévisions fiables (leur faible nombre ne permet pas de « sauver» la prévision d'une entrée erronée).

\section{IV.2. Quel rôle donner aux prévisionnistes ?}

Le bon degré de sollicitation des prévisionnistes fait l'objet de réflexions au sein des organismes comprenant des équipes opérationnelles. L'objectif est d'aller le plus loin possible dans la mise en place de systèmes automatiques proposant des premiers résultats, afin de permettre à l'être humain de : (i) se concentrer sur les étapes où il reste indispensable ou le plus utile (tant pour l'élaboration et l'expertise que pour la diffusion et la communication vers les utilisateurs avec lesquels il dialogue, cf. infra) et (ii) de produire plus rapidement des prévisions expertisées au moment où les prévisions sont les plus utiles (par exemple, tôt le matin pour diffusion via les médias). Cela suppose d'identifier de façon générale les étapes de la production où l'examen et l'intervention par un prévisionniste sont utiles, mais également les situations critiques pour lesquelles l'utilité de la prévision est la plus forte. La criticité est fonction de l'usage de la prévision (optimisation d'une production, sûreté d'un ouvrage ou gestion de crise) et de la situation particulière du jour.

À ce stade, les réflexions actuelles amènent EDF à conserver une expertise humaine pour garantir la qualité attendue au vu des enjeux de l'entreprise. De plus, les utilisateurs (internes) des prévisions veulent pouvoir contacter un « responsable » qui puisse porter et expliquer les prévisions (quel que soit le degré d'automatisation de la chaîne qui les a produites, ce qui implique une notion de « certification »).

Le réseau Vigicrues produit ses prévisions dans une perspective de gestion de crise principalement. Il a pour règle de ne pas diffuser (que ce soit au grand public ou aux gestionnaires de crise) de prévision qui n'ait pas été expertisée et validée par une prévisionniste. En crue, de très nombreux gestionnaires de crise (préfectures, etc.) s'appuient sur les explications du prévisionniste et le "degré de conviction » ressenti.

\section{IV.3. Conséquences et perspectives}

Pour faire face au besoin tout en tirant parti de l'automatisation des outils de prévision, la prévisionniste doit être capable d'adopter alternativement les deux postures identifiées par Pagano et al. [2016] : se positionner au-dessus de la chaîne de prévision automatisée ( observant ») et intervenir dans la chaîne de production (" in the loop »). Cela 
suppose de fournir au prévisionniste les outils « l'accompagnant » dans l'élaboration massive de prévisions de qualité et lui permettant d'atteindre le bon degré de cohérence. Ces outils peuvent prendre la forme de tableaux de bord et de points d'arrêt.

Les tableaux de bord sont conçus pour faciliter la supervision des prévisions automatisées par la prévisionniste en lui permettant de vérifier la qualité des données d'entrée, de contrôler la cohérence spatio-temporelle des prévisions à un instant donné et d'une exécution à l'autre des modèles, et de détecter les situations présentant le plus d'enjeux pour les partenaires. EDF et le réseau Vigicrues travaillent à la mise en place de systèmes de veille qui alerteront le prévisionniste en cas d'obsolescence d'une prévision (les observations ayant l'idée incongrue de s'éloigner de la dernière prévision expertisée) ou de changement de la situation prévue (une nouvelle prévision non expertisée s'écartant nettement de la prévision précédente), afin d'orienter la prévisionniste vers les bassins versants où son action sera la plus utile.

Les points d'arrêt ont pour objectif de permettre au prévisionniste d'être en capacité d'intervenir dans la production de prévision là où c'est nécessaire (correction des entrées, paramétrages, choix de modèles alternatifs, etc.), d'introduire son expertise dans la chaîne de production (en particulier entre un modèle amont et un modèle aval), voire de relancer l'exécution des outils de prévision.

\section{V. ÉVOLUTIONS \\ DU RÔLE DES PRÉVISIONNISTES DANS L'ACCOMPAGNEMENT DES PARTENAIRES (UTILISATEURS)}

Tant que certains utilisateurs partenaires des prévisions demanderont un accompagnement et la possibilité de dialoguer avec le prévisionniste, l'action de ce dernier dans la phase d'élaboration des prévisions doit rester suffisamment importante pour permettre une bonne appropriation des prévisions qui seront diffusées. Il s'agit en effet d'une condition nécessaire au portage efficace des prévisions vers les utilisateurs, permettant de leur expliquer les prévisions et d'en discuter les limites.

Cependant, l'augmentation du nombre de prévisions renforce la nécessité de préparer les échanges opérationnels et l'utilisation des prévisions. En effet, l'utilisation efficace d'une information plus riche (probabiliste) sur un plus grand nombre de sites, impose que la forme des prévisions diffusées soit parfaitement maîtrisée et que des outils d'aide à la décision utilisant la richesse informative de ces prévisions aient été mis en place. De même que tout changement de paradigme ${ }^{3}$ doit faire l'objet d'un accompagnement au changement permettant une appropriation sereine par les prévisionnistes, un accompagnement et des échanges préparatoires réguliers, une mise en forme standardisée des supports d'échange sont plus que jamais nécessaires au-delà de la formation initiale des partenaires, pour mettre en place et réviser les protocoles d'échanges pré-établis ( « standardisés »). L'objectif est d'éviter les temps de communication inutiles et d'assurer la cohérence de la réception des messages transmis par les centres de prévision.

3. Comme toute modification importante de la chaîne opérationnelle pour intégrer des avancées conceptuelles ou méthodologiques (par exemple, le passage des prévisions déterministes à des prévisions probabilistes).

\section{CONCLUSIONS}

Les demandes d'augmentation du nombre de prévisions hydrologiques et hydrauliques constituent un défi pour les centres de prévision qui, au-delà de la qualité de chaque prévision prise séparément, doivent s'assurer de leur cohérence « d'ensemble ». Plusieurs pistes sont d'ores et déjà identifiées pour concevoir des méthodes, des outils de prévision et des organisations humaines adaptés à cette production « de masse » de qualité et présentant une bonne cohérence spatio-temporelle. Elles demandent encore un travail important de développement, voire de recherche appliquée.

Le prévisionniste a également pour rôle de porter les prévisions vers les utilisateurs et les décideurs, que ce soit au sein d'EDF ou des services de l'État. La confiance des utilisateurs se fonde sur la prévisionniste qui explique et « assume » les prévisions et non pas sur le programme informatique ; c'est particulièrement vrai dans le contexte de la gestion de crise. Ainsi, cette évolution de la demande aura des conséquences sur le rôle du prévisionniste, au-delà du défi technique. Il est nécessaire de l'accompagner en lui permettant de concentrer ses efforts là où son intervention est la plus utile et en lui donnant des outils lui permettant d'assurer la cohérence de son expertise et de la production finale des prévisions.

Un équilibre maîtrisé entre automatisation de la production et prise en compte de l'expertise humaine doit être recherché. Cela suppose de penser conjointement le développement des outils permettant de répondre au défi de l'augmentation du nombre de prévisions à élaborer et diffuser, et le dimensionnement, la composition et la mission des équipes : le rôle dévolu au prévisionniste participe au cahier des charges pour la conception des outils et réciproquement, les outils mis en place sont dimensionnants pour le travail d'expertise.

\section{RÉFÉRENCES}

Barbetta S., Coccia G., Moramarco T., Brocca L., Todini E. (2017) - The multi temporal/multi-model approach to predictive uncertainty assessment in real-time flood forecasting. Journal of Hydrology, 551: 555-576 (doi 10.1016/j.jhydrol.2017.06.030)

Bellier J., Bontron G., Zin I. (2017) - Using Meteorological Analogues for Reordering Postprocessed Precipitations Ensembles In Hydrological Forecasting. Water Resources Research, 53: 10,085-10,107 (doi 10.1002/2017WR02145).

Bourgin F. (2014) - Comment quantifier l'incertitude prédictive en modélisation hydrologique ? Travail exploratoire sur un grand échantillon de bassins versants. AgroParisTech, thèse de doctorat. https://pastel.archives-ouvertes.fr/tel-01130084.

Courbariaux M. (2017) - Contributions statistiques aux prévisions hydrométéorologiques par méthodes d'ensemble. Université Paris - Saclay, thèse de doctorat en statistiques appliquées. https://tel.archives-ouvertes.fr/tel-01566195.

Duan Q., Pappenberger F., Thielen J., Wood A., Cloke H. L. SchAAKE J. C. (2016) - Handbook of Hydrometeorological Ensemble Forecasting, Springer Reference..

Garavaglia F., Le Lay M., Gottardi F., Garçon R., Gailhard J., PAQuet E., \& Mathevet T. (2017) - Impact of model structure on flow simulation and hydrological realism: from a lumped to a semi-distributed approach. Hydrology and Earth System Sciences, 21(8): $3937-3952$ (doi 10.5194/hess-2017-82).

KrzYszTOFowicz R. (1999) - Bayesian theory of probabilistic forecasting via deterministic hydrologic model. Water Resources Research, 35(9), 2739-2750. 
Mathevet T. (2010) - Erreur empirique de modèle. EDF - DTG : note technique interne D4165/NT/2010-00395-A.

Moulin L., Abonnel A., Puygrenier D., Valery A., Garçon R. (2018) - Prévision hydrométéorologique opérationnelle à EDF - DTG. Progrès récents et état des lieux en 2018. Colloque "De la prévision des crues à la gestion de crise " organisé par la société hydrotechnique de France (SHF). Article à paraître dans un prochain numéro de La Houille Blanche.

Pagano T. (2013) - Humans and Automation in Forecasting: International Review of the Role of Automation in River Forecasting Systems. Government of Australia - Bureau of Meteorology, technical report.

Pagano T. C., Pappenberger F., Wood A. W., Ramos M.-H., Persson A., ANDERSON B. (2016) - Automation and human expertise in operational river forecasting. WIREs Water, 3: 692-705 (doi 10.1002/wat2.1163).
Ramos M.-H., Bartholmes J., Thielen-Del Pozo J. (2007) Development of decision support products based on ensemble forecasts in the European flood alert system. Atmospheric Science Letters, 8(4): 113-119 (doi 10.1002/asl.161).

Rouhier L., Le Lay M., Garavaglia F., Le Moine N., Hendrickx F., Monteil C., Ribstein P. (2017) - Impact of mesoscale spatial variability of climatic inputs and parameters on the hydrological response. Journal of Hydrology, 553: 13-25 (doi 10.1016/j.jhydrol.2017.07.037)

Schefzik R., Thorarinsdottir T. L., Gneiting T. (2013) Uncertainty Quantification in Complex Simulation Models Using Ensemble Copula Coupling. Statistical. Science, 28(4): 616-640 (doi10.1214/13-STS443).

Viatge J., Berthet L., Marty R., Bourgin F., Piotte O., Ramos M.-H., Perrin C. (2018) - Vers une production en temps réel d'intervalles prédictifs associés aux prévisions de crue dans Vigicrues en France.. Colloque "De la prévision des crues à la gestion de crise » organisé par la société hydrotechnique de France (SHF). 